\title{
Clinicopathological characteristics of light chain proximal tubulopathy in Korean patients and the diagnostic usefulness of immunohistochemical staining for immunoglobulin light chain
}

\author{
Minsun Jung ${ }^{1}$, Youngeun Lee ${ }^{1}$, Hajeong Lee ${ }^{2,3}$ and Kyung Chul Moon ${ }^{1,3^{*}}$
}

\begin{abstract}
Background: Light chain proximal tubulopathy $(\mathrm{LCPT})$ is a rare paraproteinemic renal disease that has been mostly reported in Western patients. LCPT is characterized by the accumulation of immunoglobulin (Ig)-light chain (LC) in the proximal tubule. Immunohistochemical staining for Ig-LC has not been investigated in the context of LCPT. We reported the clinicopathological characteristics and Ig-LC immunoexpression of patients with LCPT for the first time in Korea.
\end{abstract}

Methods: We reviewed the clinicopathological findings of 5 Korean patients diagnosed with LCPT between 2016 and 2018. In addition, immunohistochemical staining for $\mathrm{k}$-LC and $\lambda$-LC was conducted on paraffin-embedded tissues.

Results: The median age was 63 years, and the male-to-female ratio was 3:2. The primary renal manifestations were either azotemia or tubular proteinuria. All patients were diagnosed with multiple myeloma with monoclonal K-LC $(\# 1-2)$ or $\lambda$-LC (\#3-5) in the serum and urine. Kidney biopsies revealed diverse and subtle alterations of the proximal tubule, including crystallization, vacuolization, and/or swelling. Electron microscopy revealed crystals in patients \#12 and non-crystalline particles within numerous/large/dysmorphic lysosomes in patients \#3-5. Ig-LC restriction was demonstrated in the proximal tubule as $\mathrm{k}$-type in patients \#1-2 and as $\lambda$-type in patients \#3-5 by immunohistochemistry and immunofluorescence. Immunohistochemical staining showed diffuse positivity to $\mathrm{k}$ and $\lambda$-LC, although immunofluorescent staining for $\mathrm{k}$-LC was focal and weak. LCPT has diverse clinicopathological characteristics and subtle morphological alterations, which necessitate ancillary tests for diagnosis.

\footnotetext{
* Correspondence: blue7270@snu.ac.kr

'Department of Pathology, Seoul National University Hospital, Seoul 03080,

Republic of Korea

${ }^{3}$ Kidney Research Institute, Medical Research Center, Seoul National

University College of Medicine, Seoul 03080, Republic of Korea

Full list of author information is available at the end of the article
}

(c) The Author(s). 2020 Open Access This article is licensed under a Creative Commons Attribution 4.0 International License, which permits use, sharing, adaptation, distribution and reproduction in any medium or format, as long as you give appropriate credit to the original author(s) and the source, provide a link to the Creative Commons licence, and indicate if changes were made. The images or other third party material in this article are included in the article's Creative Commons licence, unless indicated otherwise in a credit line to the material. If material is not included in the article's Creative Commons licence and your intended use is not permitted by statutory regulation or exceeds the permitted use, you will need to obtain permission directly from the copyright holder. To view a copy of this licence, visit http://creativecommons.org/licenses/by/4.0/ The Creative Commons Public Domain Dedication waiver (http://creativecommons.org/publicdomain/zero/1.0/) applies to the data made available in this article, unless otherwise stated in a credit line to the data. 
(Continued from previous page)

Conclusions: We introduced immunohistochemical staining for lg-LC as a useful tool for the diagnosis of LCPT, especially in the case of k-type crystals.

Keywords: Immunoglobulin light chains, Immunohistochemistry, Light chain proximal Tubulopathy, Monoclonal Gammopathy, Multiple myeloma

\section{Background}

Monoclonal light chain (LC), when overproduced in multiple myeloma (MM) and other paraproteinemic hematologic disorders, can affect the renal tubule [1]. For example, monoclonal immunoglobulin (Ig)-LC precipitates with Tamm-Horsfall protein in the distal tubule of the kidney, resulting in cast nephropathy which is the most common renal disorder of MM [1,2]. Recently, light chain proximal tubulopathy (LCPT) has been emphasized as a distinct entity of LC-related kidney diseases. LCPT has been typified by the crystallization of $\mathrm{K}-$ $\mathrm{LC}$ in the proximal tubule, which is associated with Fanconi syndrome in approximately $40 \%$ of patients $[2,3]$. LCPT without crystal, which was described more recently, is associated with either $\mathrm{k}$ - or $\lambda$-LC accumulation $[2,4]$. LCPT can be an early manifestation of an underlying hematologic disorder [2, 5-7], and prompt treatment can prevent permanent renal impairment $[2,8]$; therefore, it is important to properly diagnose LCPT. However, the clinicopathological characteristics of LCPT are not fully defined, which makes the diagnosis challenging [7]. Moreover, although immunofluorescence (IF) and electron microscopy (EM) are frequently utilized in the diagnosis process, immunohistochemical (IHC) staining for Ig-LC has not been investigated in the context of LCPT. Here, we report the clinicopathological characteristics of 5 Korean patients with LCPT and describe the diagnostic implications of IHC staining for IgLC.

\section{Methods}

We retrieved 5 kidney biopsy samples from patients diagnosed with LCPT at Seoul National University Hospital between 2016 and 2018. Any patients who showed evidence of paraprotein-induced glomerular disease, amyloidosis, or cast nephropathy were excluded $[1,4,7]$. All specimens were routinely evaluated using light microscopy (LM), IF, and EM. In addition, IHC staining for к-LC (1:500; L1C1; Leica, Wetzlar, Germany) and $\lambda$-LC (1:1500; HP-6054; Leica) was conducted on paraffinembedded tissues using the Benchmark autostainer (Ventana, Tucson, AZ) following the manufacturer's instructions. The extent of histologic alterations in the proximal tubule, including acute tubular injury (ATI) and interstitial fibrosis and tubular atrophy (IFTA), was measured based on LM as absent, present but minimal
$(<10 \%)$, or present $(>10 \%)$. The IF and IHC staining results were evaluated as either focal $(<50 \%)$ or diffuse $(\geq 50 \%)$. Laboratory data and follow-up information were obtained from medical records. The median follow-up period was 20 months (range, 4-24 months). Biopsies were performed within 1 month after the detection of monoclonal gammopathy. The diagnosis and treatment response of MM were based on standard criteria $[9,10]$. MM stage was evaluated according to the International Staging System (ISS) [11].

\section{Results}

The clinical features of patients with LCPT are summarized in Table 1. The patients' ages ranged from 51 to 74 years. Three patients were men and two were women. Patients \#1 and \#3-4 had preexisting hypertension, and patients \#3-4 had diabetes mellitus. The primary renal manifestations were either azotemia in 3 patients or tubular proteinuria in 3 patients, both of which were present in patient \#1 (Table 1). Features of Fanconi syndrome, including glycosuria, hypouricemia, hypophosphatemia, polyuria, acidosis, and hypokalemia, were not observed in any patients. In the hematologic evaluation, all patients were diagnosed with MM in ISS stage I (\#3, $5)$ or II $(\# 1,4)$ (Table 1$)$. Monoclonal Ig-LC was identified in the serum and urine as $\mathrm{k}(\# 1-2)$ or $\lambda(\# 3-5)$ with an increased or decreased $\kappa-$ to- $\lambda$ free LC ratio, respectively. All patients received combined chemotherapeutic regimens. Autologous stem cell transplantation was followed in patients \#2 and 4. At the last check-up, complete $(\# 2,4)$ or partial $(\# 1,3,5)$ hematologic remission was achieved, and all patients exhibited normalization/improvement of renal function. One patient (\#3) died of unknown causes.

The pathological characteristics of the kidney biopsies are summarized in Table 2, Fig. 1, and Fig. 2. The proximal tubule was predominantly affected in all patients, exhibiting vacuolated and/or swollen cytoplasm with a glassy texture that was diffuse (\#1-4) or focal (\#5), as observed with LM (Fig. 1a, Fig. 2a). In addition, fuchsinophilic crystals were focally observed in patient \#1 (Fig. $1 \mathrm{~b})$. Identical types of monoclonal Ig-LC in the system were detected by IF and IHC staining as follows: $\mathrm{k}$-type in patients $\# 1-2$, and $\lambda$-type in patients \#3-5. The immunoreaction was focal for $\mathrm{k}$-LC (Fig. 1c) but diffuse for $\lambda$-LC (Fig. 2c) by IF; in contrast, diffuse Ig-LC restriction 
Table 1 Clinical features and treatment outcomes of patients with light chain proximal tubulopathy

\begin{tabular}{|c|c|c|c|c|c|}
\hline & Case \#1 & Case \#2 & Case \#3 & Case \#4 & Case \#5 \\
\hline Renal presentation & Azotemia and proteinuria & Azotemia & Azotemia & Proteinuria & Proteinuria \\
\hline \multicolumn{6}{|l|}{ Renal baseline } \\
\hline $\mathrm{SCr}(\mathrm{mg} / \mathrm{dl})$ & 2.10 & 1.93 & 1.88 & 1.06 & 1.00 \\
\hline $\mathrm{eGFR}^{\mathrm{a}}\left(\mathrm{ml} / \mathrm{min} / 1.73 \mathrm{~m}^{2}\right)$ & 31.0 & 27.3 & 35.5 & 52.5 & 75.3 \\
\hline UPCr & 3.64 & 1.64 & 0.89 & 4.26 & 1.39 \\
\hline Hematologic Dx & MM & MM & MM & MM & MM \\
\hline BM plasma cell (\%) & 13.2 & 66.5 & 47.3 & 11.4 & 11.0 \\
\hline ISS & $\|$ & n.a. & 1 & $\|$ & । \\
\hline Serum $\beta 2$-microglobulin $(\mu \mathrm{g} / \mathrm{ml})$ & 4.70 & n.a. & 3.01 & 4.26 & 2.79 \\
\hline Urine $\beta 2$-microglobulin $(\mu \mathrm{g} / \mathrm{ml})$ & 5.48 & n.a. & 0.61 & 0.46 & n.a. \\
\hline Monoclonal lg & K & $G-k$ & $\lambda$ & $G-\lambda$ & $A-\lambda$ \\
\hline Serum $k / \lambda$ ratio $^{b}$ & 193.43 & 37.61 & 0.01 & $<0.01$ & 0.01 \\
\hline Urine $k / \lambda$ ratio $^{c}$ & 1245.65 & 284.08 & 0.11 & $<0.01$ & n.a. \\
\hline$F S^{d}$ & Absent & Absent & Absent & Absent & Absent \\
\hline Serum uric acid (mg/dl) & 5.4 & 7.9 & 8.4 & 3.6 & 8.6 \\
\hline Serum phosphorus (mg/dl) & 3.2 & 3.6 & 4.2 & 3.7 & 3.8 \\
\hline Treatment & VMP\#9 & VTD\#5 $\rightarrow$ SCT & VMP\#2 & VTD\#3 $\rightarrow$ SCT & VTD\#5 \\
\hline Last f/u (month) & 20 & 24 & 4 & 20 & 6 \\
\hline Hematologic outcome & $P R$ & $C R$ & $P R$ & $C R$ & $P R$ \\
\hline \multicolumn{6}{|l|}{ Renal outcome } \\
\hline $\mathrm{SCr}(\mathrm{mg} / \mathrm{dl})$ & 1.26 (WNL) & 0.72 (WNL) & 1.44 (improved) & 0.97 (WNL) & 0.87 (WNL) \\
\hline $\mathrm{eGFR}^{\mathrm{a}}\left(\mathrm{ml} / \mathrm{min} / 1.73 \mathrm{~m}^{2}\right)$ & 55.7 (WNL) & 84.7 (WNL) & 48.2 (improved) & $58.0(\mathrm{WNL})$ & 88.3 (WNL) \\
\hline UPCr & 0.23 (improved) & $<0.2$ (WNL) & $<0.2$ (WNL) & $<0.2$ (WNL) & 0.25 (improved) \\
\hline
\end{tabular}

Based on Modification of Diet in Renal Disease Study equation

${ }^{\text {b Normal range: } 0.26-1.65}$

'Normal range: $2.04-10.37$

${ }^{\mathrm{d}} \mathrm{Glycosuria}$, hypouricemia, hypophosphatemia, polyuria, acidosis, and hypokalemia

$M$ male, $F$ female, $S C r$ serum creatinine, eGFR estimated glomerular filtration rate, UPCr urine protein/creatinine ratio, Dx diagnosis, $M M$ multiple myeloma, $B M$ bone marrow, ISS International Staging System for Multiple Myeloma, n.a. not available, $I g$ immunoglobulin, FS Fanconi syndrome, VMP Velcade, Melphalan, prednisone, VTD Velcade, thalidomide, dexamethasone, SCT autologous stem-cell transplantation, f/u follow-up, PR partial remission, CR complete remission, WNL within normal limits

was detected by IHC in all patients (Fig. 1d, Fig. 2d). When observed with EM, the accumulated Ig-LC appeared as rhomboid-like (\#1; Fig. 1e) or needle-like (\#2; Fig. 1f) crystals in LCPT with crystal or as electron-dense particles in the lysosome in LCPT without crystal, which led to a mottled appearance (\#3-5; Fig. 2e, f). Although similar deposits were occasionally observed in patients \#1-2 (Fig. 1e), the lysosomes of patients \#3-5 were numerous, large, and frequently dysmorphic (Fig. 2e, f). In addition to these changes, ATI (Fig. 2b) and IFTA were present in 5 and 4 patients, respectively. Luminal proteinaceous material (Fig. 1a) was present in varying degrees in 4 patients, and this material was reactive to both $\mathrm{k}$ - and $\lambda$-LCs with similar intensity on IHC staining. The glomeruli and blood vessels were unremarkable, except for occasional global sclerosis and mild fibrointimal thickening, respectively.

\section{Discussion}

We delineated the clinicopathological characteristics of patients with LCPT for the first time in Korea. LCPT accounted for $0.1-1.5 \%$ of native renal biopsies according to retrospective studies [4, 7]. Approximately 200 cases of LCPT have been reported, most of which have been from Western countries, with a small number of patients from Asian countries; LCPT has not been described in Korean patients $[2-4,7,12-16]$. Various plasma cell dyscrasias, such as MM in this report, can lead to the overproduction of free LCs, which are filtered by the glomeruli and reabsorbed in the proximal tubule $[1,17]$. An accumulation of Ig-LC in the proximal tubule that exceeds the capacity of lysosomal proteolysis results in LCPT, and the chemodynamics of this Ig-LC are known to determine the clinicopathological features of LCPT $[1,7]$. Thus, the detection of Ig-LC restriction in the proximal tubule is critical for the diagnosis of LCPT 
Table 2 Pathological characteristics of patients with light chain proximal tubulopathy

\begin{tabular}{|c|c|c|c|c|c|}
\hline & Case \#1 & Case \#2 & Case \#3 & Case \#4 & Case \#5 \\
\hline \multicolumn{6}{|l|}{ PTEC } \\
\hline LM & $\begin{array}{l}\text { Focal fuchsinophilic } \\
\text { crystal, diffuse } \\
\text { vacuolization and } \\
\text { swelling }\end{array}$ & $\begin{array}{l}\text { Focal vacuolization and } \\
\text { diffuse swelling }\end{array}$ & $\begin{array}{l}\text { Diffuse vacuolization and } \\
\text { swelling }\end{array}$ & Diffuse vacuolization & Focal swelling \\
\hline $\lg -\mathrm{LC}$ & k & k & $\lambda$ & $\lambda$ & $\lambda$ \\
\hline $\mathbb{F}^{\mathrm{a}}$ & Focal & Focal & Diffuse & Diffuse & Diffuse \\
\hline $\mathrm{HC}^{\mathrm{a}}$ & Diffuse & Diffuse & Diffuse & Diffuse & Diffuse \\
\hline EM & $\begin{array}{l}\text { Crystal (rhomboid), } \\
\text { lysosome with EDD }\end{array}$ & $\begin{array}{l}\text { Crystal (needle-like), } \\
\text { lysosome with EDD and } \\
\text { focal dysmorphism }\end{array}$ & $\begin{array}{l}\text { Increased/large/ } \\
\text { dysmorphic lysosome } \\
\text { with mottled appearance }\end{array}$ & $\begin{array}{l}\text { Increased/large/ } \\
\text { dysmorphic lysosome } \\
\text { with mottled appearance }\end{array}$ & $\begin{array}{l}\text { Increased/large/ } \\
\text { dysmorphic lysosome } \\
\text { with mottled appearance }\end{array}$ \\
\hline$A T I^{b}$ & Present, minimal & Present, minimal & Present, minimal & Present & Present, minimal \\
\hline IFTA $A^{b}$ & Present, minimal & Present, minimal & Present & Present & Absent \\
\hline $\begin{array}{l}\text { Tubular } \\
\text { proteinaceous } \\
\text { material }\end{array}$ & Present & Present & Absent & Present & Present \\
\hline Glomerulus & GS (12.1\%) & WNL & GS (12.5\%) & GS (18.6\%) & WNL \\
\hline Blood vessel & Fibro-intimal thickening & WNL & Fibro-intimal thickening & Fibro-intimal thickening & Fibro-intimal thickening \\
\hline
\end{tabular}

${ }^{\mathrm{a}}$ Focal IF or IHC staining: positivity in $<50 \%$ of the proximal tubule

${ }^{\mathrm{b}}$ Minimal ATI or IFTA: $0-10 \%$ of the renal cortex

PTEC Proximal tubule epithelial cell, LM Light microscopy, Ig-LC Immunoglobulin light chain, IF Immunofluorescence, IHC Immunohistochemistry, EM Electron microscopy, EDD Electron-dense deposit, ATI Acute tubular injury, IFTA Interstitial fibrosis with tubular atrophy, GS Global sclerosis, WNL Within normal limit

[7]. We demonstrated that IHC staining for Ig-LC was strongly positive for the identical Ig-LC detected by IF and even for $\mathrm{k}$-LC, which exhibited a limited reaction by IF. By forming a crystalline structure, $\mathrm{K}$-LC may evade immunoreaction in IF staining [7]. Although pronase treatment of the paraffin block has been suggested to enhance the immunoreactivity of IF staining for $\mathrm{k}$-type $\mathrm{LC}$, this treatment is not always successful $[7,18]$. Furthermore, immunogold analysis, which would be confirmative, is not widely available $[7,12]$. The antigen
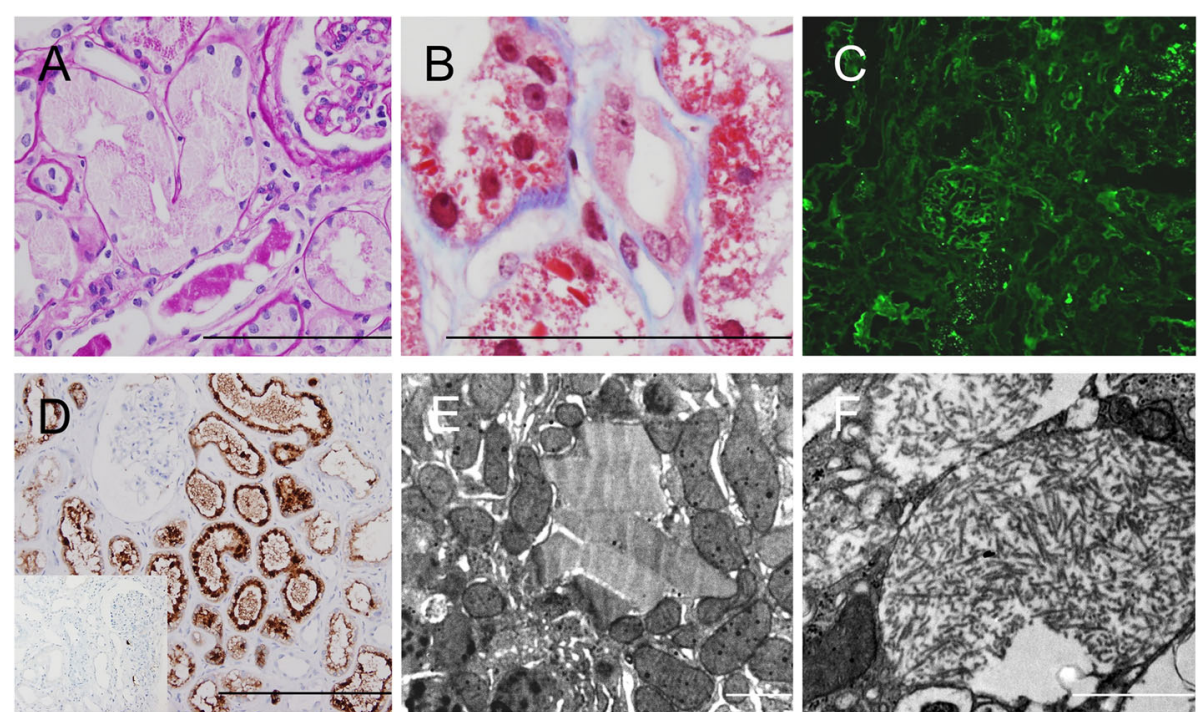

Fig. 1 Microscopic findings of LCPT with crystal. a LCPT with crystal shows swollen tubules with abundant glassy cytoplasm along with an injured tubule that contains proteinaceous material in the lumen in patient \#2 (PAS). b In addition, fuchsinophilic crystals are observed in the vacuolar cytoplasm of the tubules in patient \#1 (MT). $\mathrm{K}-\mathrm{LC}$ is restricted in the tubular cytoplasm which is (c) weak and focal by IF but (d) diffuse by IHC ( $\lambda$-LC in an inlet) staining in patient \#1. EM analysis demonstrates $(\mathbf{e}, \# 1)$ rhomboid or (f, \#2) needle-like crystals in the tubular cytoplasm with (e) occasional electron-dense deposits in the lysosome (left bottom). Scale bars indicate $10 \mu \mathrm{m}$ in LM and $1 \mu \mathrm{m}$ in EM figures 

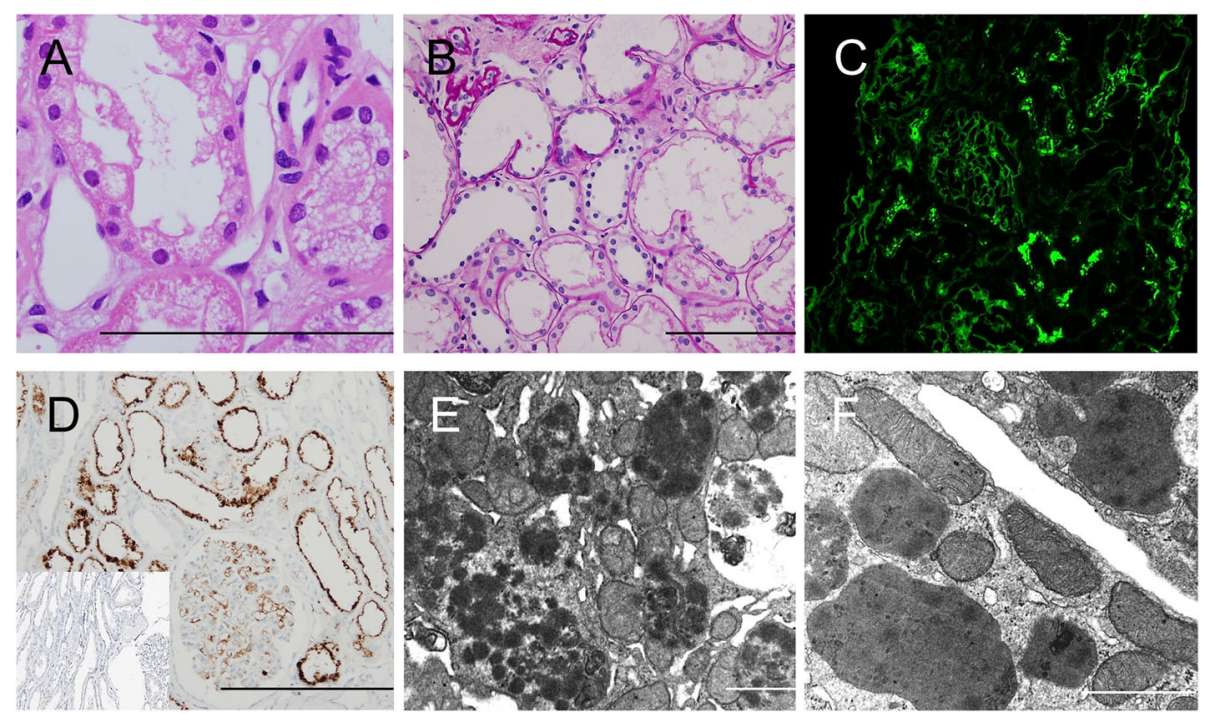

Fig. 2 Microscopic findings of LCPT without crystal. LCPT without crystal shows (a) vacuolization (\#3, HE) and (b) diffuse damage (\#4; PAS) of the tubule. Diffuse accumulation of $\lambda-\mathrm{LC}$ is observed both in (c) IF and (d) IHC ( $\mathrm{K}-\mathrm{LC}$ in an inlet) staining in patient \#5. e The lysosome in the tubular cytoplasm is increased in number and contains numerous electron-dense deposits, which leads to mottled appearance in patient \#4. $\mathbf{f}$ Large dysmorphic lysosomes with electron-dense particles are seen in patient \#5. Scale bars indicate $10 \mu \mathrm{m}$ in LM and $1 \mu \mathrm{m}$ in EM figures

retrieval step during IHC staining may enhance the immunoreactivity of Ig-LC. Therefore, we propose IHC staining for Ig-LC as a useful diagnostic tool for LCPT that would be complementary to or even a substitution for IF in patients with weak or inconclusive IF results.

This and previous reports on LCPT described tubulecentered pathological findings $[2,4,7]$. We detected fuchsinophilic crystals that were focally present in one of the two patients with LCPT with crystal; such crystals were previously reported to be observed with varying shapes and in varying degrees by LM in crystalline LCPT [2]. The presence of rhomboid-like or needle-like crystals under EM can confirm the diagnosis. Prior studies described the following variable histological features of LCPT without crystal: normal [4], cytoplasmic vacuolization $[2,7]$ or swelling $[4,7]$, tubular injury $[2,4,7]$, or even necrosis [7]. In all patients in the present study, swelling and/or vacuolization of the proximal tubule were observed at least focally and were more apparent in crystalline LCPT than in non-crystalline LCPT [3]. In both types of LCPT, however, these morphological findings were generally subtle and easy to miss, which we ascribed to the promptness of systemic and renal workups. Therefore, a high degree of suspicion during the microscopic analyses and meticulous ancillary tests, including IF/IHC and EM, are required for the diagnosis [5]. The detection of numerous and sometimes dysmorphic lysosomes with a mottled appearance via EM was essential to diagnose LCPT without crystal in this and previous studies [2, 4, 7]. However, similar ultrastructural findings are shared by patients with LCPT with crystal to a lesser degree, which suggests that this feature is not entirely specific to LCPT without crystal. Moreover, these EM findings of the lysosome have been inconsistently included in the diagnostic criteria of LCPT without crystal in previous studies, which may explain the inconsistently reported proportion of noncrystalline LCPT, ranging from 13 to $77 \%[2,4,7,8]$. Because of the rarity and diverse pathological features of LCPT without crystal, the standardization of its diagnosis may warrant further investigations.

Consistent with prior studies $[2,4,8,12]$, renal insufficiency and/or tubular proteinuria were the main signs of LCPT, and they appeared to be similar between patients with crystalline and non-crystalline forms of LCPT. Signs of Fanconi syndrome were not observed in any patients. Although Stokes et al. suggested that patients with LCPT with crystal may have worse renal function and proteinuria and closer association with Fanconi syndrome than those with LCPT without crystal [2], we did not find such trends, which can be ascribed to the small number of patients in our study. The extent of ATI and IFTA was unlikely to be associated with initial and posttreatment renal function in this and previous reports [2]. Prompt and accurate diagnosis of LCPT was suggested to be important to prevent irreversible kidney dysfunction in patients with monoclonal gammopathy $[1,17]$. MM therapy considerably improved the kidney function of patients with LCPT in the present report and previous reports $[2,8]$. Luminal proteinaceous material, which was presumably an organized Ig-LC released from desquamated tubular epithelial cells $[2,3]$, can be a 
diagnostic pitfall. This material was differentiated from that of cast nephropathy by its location and the lack of multiple fracture lines or giant cell reaction [1]. Furthermore, it is reasonable to speculate that pathogenic LCPT without crystal needs to be differentiated from the physiological trafficking of overflowing LC with a benign nature $[2,4,17]$.

\section{Conclusions}

We reported the clinicopathological characteristics of 5 Korean patients with LCPT with or without crystal. The tubule-oriented microscopic features of LCPT are diverse and sometimes subtle. We introduced IHC staining for Ig-LC as a useful tool in the diagnosis of LCPT, especially in the case of $\kappa$-type crystals.

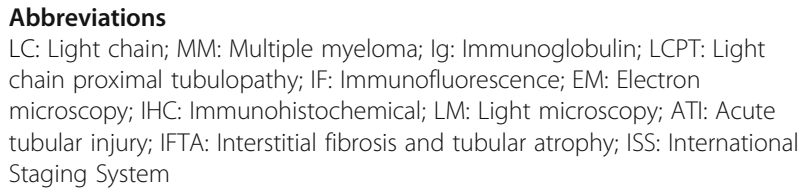

\section{Acknowledgements}

Not applicable.

\section{Authors' contributions}

KCM designed the ancillary study. MJ and YL performed the histological examination of the kidney. HL analyzed and interpreted the clinical data. MJ was a major contributor in writing the manuscript. All authors read and approved the final manuscript.

\section{Funding}

This research received no external funding.

\section{Availability of data and materials}

All data generated or analysed during this study are included in this published article.

\section{Ethics approval and consent to participate}

All procedures were approved by institutional review board of Seoul National University Hospital (H-1805-054-944). All research was performed in accordance with the relevant guidelines and regulations. Informed consent was waived by the review board due to very low risk to the subject.

\section{Consent for publication}

Not applicable.

\section{Competing interests}

The authors declare that they have no competing interests.

\section{Author details \\ 'Department of Pathology, Seoul National University Hospital, Seoul 03080, Republic of Korea. ${ }^{2}$ Department of Internal Medicine, Seoul National University Hospital, Seoul 03080, Republic of Korea. ${ }^{3}$ Kidney Research Institute, Medical Research Center, Seoul National University College of Medicine, Seoul 03080, Republic of Korea.}

Received: 22 August 2019 Accepted: 16 April 2020

Published online: 23 April 2020

\section{References}

1. Doshi M, Lahoti A, Danesh FR, Batuman V, Sanders PW. Paraprotein-related kidney disease: kidney injury from paraproteins-what determines the site of injury? Clin J Am Soc Nephrol. 2016;11:2288-94.
2. Stokes $M B$, Valeri $A M$, Herlitz L, et al. Light chain proximal tubulopathy: clinical and pathologic characteristics in the modern treatment era. J Am Soc Nephrol. 2016;27:1555-65.

3. Messiaen T, Deret $\mathrm{S}$, Mougenot $\mathrm{B}$, et al. Adult fanconi syndrome secondary to light chain gammopathy. Clinicopathologic heterogeneity and unusual features in 11 patients. Medicine (Baltimore). 2000;79:135-54.

4. Larsen CP, Bell JM, Harris AA, Messias NC, Wang YH, Walker PD. The morphologic spectrum and clinical significance of light chain proximal tubulopathy with and without crystal formation. Mod Pathol. 2011;24:14629.

5. Kapur U, Barton K, Fresco R, Leehey DJ, Picken MM. Expanding the pathologic spectrum of immunoglobulin light chain proximal tubulopathy. Arch Pathol Lab Med. 2007;131:1368-72.

6. Zakharova EV, Stolyarevich ES, Vorobyeva OA, Nikitin EA. Combined immunoglobulin g kappa nephropathy: monoclonal immunoglobulin deposition disease and proximal tubulopathy: monoclonal gammopathy of renal significance or smoldering multiple myeloma? Case report and review of literature. Integr Cancer Sci Therap. 2017;4:1-9.

7. Herrera GA. Proximal tubulopathies associated with monoclonal light chains: the spectrum of clinicopathologic manifestations and molecular pathogenesis. Arch Pathol Lab Med. 2014;138:1365-80.

8. Vignon M, Javaugue V, Alexander MP, et al. Current anti-myeloma therapies in renal manifestations of monoclonal light chain-associated fanconi syndrome: a retrospective series of 49 patients. Leukemia. 2017:31:123-9.

9. Rajkumar SV, Dimopoulos MA, Palumbo A, et al. International myeloma working group updated criteria for the diagnosis of multiple myeloma. Lancet Oncol. 2014;15:e538-48.

10. Kumar S, Paiva B, Anderson KC, et al. International myeloma working group consensus criteria for response and minimal residual disease assessment in multiple myeloma. Lancet Oncol. 2016;17:e328-e46.

11. Palumbo A, Avet-Loiseau H, Oliva S, et al. Revised international staging system for multiple myeloma: a report from international myeloma working group. J Clin Oncol. 2015;33:2863-9.

12. Sharma SG, Bonsib SM, Portilla D, Shukla A, Woodruff AB, Gokden N. Light chain proximal tubulopathy: expanding the pathologic spectrum with and without deposition of crystalline inclusions. ISRN Pathol. 2012. https://doi. org/10.5402/2012/541075.

13. Noguchi K, Munemura C, Maeda S, et al. Myeloma-associated fanconi syndrome due to $\lambda$-light chain crystal deposition. Yonago Acta Med. 2004; 47:91-6.

14. Yu XJ, Zhang X, Li DY, Wang SX, Zhou FD, Zhao MH. Renal pathologic spectrum and clinical outcome of monoclonal gammopathy of renal significance: a large retrospective case series study from a single institute in China. Nephrology (Carlton). 2020;25:202-11.

15. Kawamoto S, Hidaka Y, Kaneko Y, et al. Remission of light chain proximal tubulopathy in igg lambda-type multiple myeloma by lenalidomide and dexamethasone therapy. CEN Case Rep. 2019;8:159-65.

16. Kishi S, Obata F, Miki $H$, et al. Lambda light chain non-crystalline proximal tubulopathy with igd lambda myeloma. Intern Med. 2018;57:3597-602.

17. Bridoux F, Leung N, Hutchison CA, et al. Diagnosis of monoclonal gammopathy of renal significance. Kidney Int. 2015;87:698-711.

18. Nasr SH, Galgano SJ, Markowitz GS, Stokes MB, D'Agati VD. Immunofluorescence on pronase-digested paraffin sections: a valuable salvage technique for renal biopsies. Kidney Int. 2006;70:2148-51.

\section{Publisher's Note}

Springer Nature remains neutral with regard to jurisdictional claims in published maps and institutional affiliations. 